\title{
Particle simulation study of electron heating by counterstreaming ion beams ahead of supernova remnant shocks
}

\author{
M E Dieckmann ${ }^{1}$, A Bret ${ }^{2,3}$, G Sarri $^{1}$, E Perez Alvaro ${ }^{3}$, I \\ Kourakis $^{1}$ and M Borghesi ${ }^{1}$ \\ 1. Queen's University Belfast, Ctr Plasma Phys, Belfast BT7 1NN, UK \\ 2. Harvard-Smithsonian Center for Astrophysics, Cambridge, MA 02138, USA \\ 3. ETSI Industriales, Universidad de Castilla-La Mancha, 13071 Ciudad Real, Spain \\ and Instituto de Investigaciones Energeticas y Aplicaciones Industriales, Campus \\ Universitario de Ciudad Real, 13071 Ciudad Real, Spain
}

\begin{abstract}
The growth and saturation of Buneman-type instabilities is examined with a particle-in-cell (PIC) simulation for parameters that are representative for the foreshock region of fast supernova remnant (SNR) shocks. A dense ion beam and the electrons correspond to the upstream plasma and a fast ion beam to the shock-reflected ions. The purpose of the $2 \mathrm{D}$ simulation is to identify the nonlinear saturation mechanisms, the electron heating and potential secondary instabilities that arise from anisotropic electron heating and result in the growth of magnetic fields. We confirm that the instabilities between both ion beams and the electrons saturate by the formation of phase space holes by the beam-aligned modes. The slower oblique modes accelerate some electrons, but they can not heat up the electrons significantly before they are trapped by the faster beam-aligned modes. Two circular electron velocity distributions develop, which are centred around the velocity of each ion beam. They develop due to the scattering of the electrons by the electrostatic wave potentials. The growth of magnetic fields is observed, but their amplitude remains low.
\end{abstract}

PACS numbers: 52.35.Qz, 52.50.Gj, 52.65.Rr 


\section{Introduction}

The thermalisation of shock-reflected ion beams plays an important role in solar system and astrophysical collision-less plasma. Energetic beams, which consist of the ions that were reflected by a plasma shock, outrun the shock and interact with the upstream plasma. This upstream plasma is the interstellar medium (ISM) for supernova remnant (SNR) shocks and the solar wind for most solar system shocks. The ion beam is a source of free energy, which is released through wave instabilities. Such instabilities have been observed ahead of solar system shocks [1]. They are also important mechanisms for particle acceleration at supernova remnant (SNR) shocks [2-9].

The Buneman instability [10, 11] develops between one ion beam and one electron beam of equal density, which are both spatially uniform, unmagnetized and drift relative to each other. Such a plasma is not current neutral. A plasma with no net charge and no net current can be composed of two counter-streaming ion beams and one electron species, if the total charge density of the ions is that of the electrons and if the partial currents of the three beams cancel out each other. This can be an appropriate description of the plasma upstream of a shock. One ion beam is composed of the shock-reflected ions and the second ion beam and the electrons are provided by the upstream plasma, into which the shock expands. Such distributions are observed ahead of the Earth's bow shock [1]. We refer with Buneman-type instability (BTI) to an electrostatic instability, which involves an ion beam with a density below that of the electrons.

A BTI develops, if the drift speed between the ion beam and the electrons exceeds the electron thermal speed. Thermal damping effects due to the ions can be neglected, as long as they are reasonably cold. If the shock-reflected ion beam is much faster than the electron thermal speed and sufficiently dense, then its current has to be cancelled out by a high drift speed between the background electrons and ions. This drift speed may exceed the electron thermal speed and result in a second BTI. The speed of SNR shocks is a few per cent of the light speed c, which exceeds by far the electron thermal speed in the ISM. We may expect that the shock-reflected ion beam and the counterstreaming ion beam are both sufficiently fast in the electron rest frame to render the plasma unstable. Such plasmas have been examined widely in the past with particle-in-cell (PIC) simulations [12. Previous studies have addressed unmagnetized [13, 14, 15] and magnetized plasmas [16, 17] with one-dimensional PIC simulations, which can not capture the multi-dimensional nature of the wave fields. More recently, the BTI has been examined with two-dimensional PIC simulations in unmagnetized and magnetized plasma. The electrostatic simulation in Ref. [18] has modeled the interaction of one fast ion beam with cool electrons. References [19, 20] investigated ion beams that stream with nonrelativistic and mildly relativistic speeds across an orthogonal magnetic field.

We examine here with an electromagnetic 2D PIC simulation how a plasma thermalises, which consists of two counterstreaming ion beams with unequal densities that move through an unmagnetized electron plasma. The drift speeds between the electrons and each of the ion beams exceed the initial electron thermal speed by factors 
of 3.4 and 17 and two BTI's develop. The faster beam has a speed that is comparable to the ones modeled with PIC simulations in Ref. [18]. The waves driven by both BTI's interact nonlinearly with the electrons, once their amplitude is adequate. Trapping by an electrostatic wave accelerates electrons along the wave vector and a thermal anisotropy in the electron's velocity distribution develops as we show here. It is the purpose of our study to assess, if a thermal anisotropy-driven Weibel instability (TAWI) [21-29] is triggered by anisotropic electron heating. Such a secondary instability can not be resolved by the electrostatic PIC code used in Ref. [18].

The initial conditions, which we consider here, are representative for a volume element in the foreshock, that is so small that the flow speed of the upstream plasma and of the shock-reflected ion beam are constant inside. However, ion beam-driven instabilities can also be triggered by the return current [30] of the cosmic rays. Similar instabilities may thus occur in a much larger volume ahead of the shock. The TAWI could provide [31] the seed magnetic field, which speeds up the cosmic ray-driven instabilities that magnetize SNR shocks [32, 33]. The inital magnetic amplitude is set to zero in our simulation, which simplifies the detection and interpretation of magnetic fields that are driven by the BTI through the subsequent TAWI. This choice can be justified by the weak magnetic fields ahead of SNR shocks, which yield an electron cyclotron frequency well below the BTI's frequency in the electron rest frame. At the same time, the magnetic field close to SNR shocks is thought to be strong enough to reflect enough ions to form a dense beam, provided that the shock is perpendicular.

Our results are as follows. The interaction of the background electrons with the slow and dense ion beam from the background plasma yields the faster-growing instability, as we expect from the solution of the linear dispersion relation. The electrostatic waves grow and saturate through the formation of electron phase space tubes [34-39]. The spectrum of the unstable waves is not unidirectional [18] and the phase space tubes are spatially bounded. Their extent orthogonal to their wave vector is a few times their wave length. Trapping accelerates the electrons into the direction of the propagating waves. The electron thermal energy along the beam direction reaches twice the value of the electron thermal energy orthogonal to the beam direction and this anisotropy results in the growth of magnetic fields. The magnetic fields do, however, only reach an energy density that is 2-3 orders of magnitude below that of the electric fields and their strength does not exceed that of the interstellar magnetic fields.

The electrons are initially accelerated by trapping to a speed, which is about twice as high as the background ion speed in the electron rest frame. After this initial acceleration, the electrons are scattered by the waves. The reflections are elastic, because the electrostatic wave potential is almost time-stationary in the reference frame of the bulk ions, and they change the momentum direction of the electrons. Repeated collisions between the electrons and the phase space tubes redistribute the electrons into a circular interval in velocity space, which is centered at the phase speed of the waves. This mechanism differs subtly from that proposed in Ref. [18], which has attributed the crescent formation to the low phase speed of the oblique modes. 
The plasma is no longer free of current after the saturation of the first BTI. The electrons have been decelerated in the rest frame of the background ions by their interaction with the waves while the ions with their high inertia have only been weakly affected. A macroscopic beam-aligned electrostatic field grows in response to this current on scales much larger than the wave length of the BTI [40]. This field accelerates the electrons nonresonantly such that the mean electron speed remains close to the one prior to the wave saturation, thereby reducing the net current.

Eventually the electrons start to interact with the faster ion beam. Electrons are accelerated primarily by trapping, but the Landau damping of the oblique modes results in a separate population of hot electrons. A crescent distribution develops, but it is less pronounced than the one found in Ref. [18]. The electron heating by the first BTI and the electron acceleration by the macroscopic electric field have changed the electron distribution function to a non-Maxwellian one and they have decreased the ratio between beam speed and electron thermal speed to a value well below that used in Ref. [18, which may explain the different plasma evolution. The hotter ion beam we consider here will also modify the wave spectrum of the BTI and the nonlinear response of the beam ions to the electric fields, which may also contribute to the different interaction of the electrons with the waves driven by the second BTI. An energetic circular distribution in velocity space gradually develops after the second BTI has saturated by electron trapping. The cause is again the electron scattering by the electrostatic structures that move now with the mean velocity of the fast ion beam. No TAWI develops in this case during the simulation time, even though the electrons continue to show a thermal anisotropy. The absence of any significant magnetic field growth during the growth and saturation of the two BTIs suggests that the thermal anisotropy created by the nonlinear interaction between electrostatic waves and electrons is not capable of magnetising the foreshock of supernova remnant shocks, at least not for our initial conditions.

The structure of the paper is as follows. Section 2 discusses the initial conditions and the PIC simulation code. Section 3 presents the simulation results. Section 4 is the discussion.

\section{The initial conditions and the particle-in-cell simulation code}

\subsection{Initial conditions}

We consider here three spatially uniform plasma species with non-relativistic Maxwellian velocity distributions. The electrons have the density $n_{0}$, the plasma frequency

$\omega_{p}=\left(n_{0} e^{2} / m_{e} \epsilon_{0}\right)^{1 / 2}$ and the temperature $10 \mathrm{eV}$ or the thermal speed $v_{t e}=1.325 \times 10^{6}$ $\mathrm{m} / \mathrm{s}$. They move at the speed $v_{e}=4.5 \times 10^{6} \mathrm{~m} / \mathrm{s}$ along increasing values of $x$, which gives $v_{e}=3.4 v_{t e}$. We consider for computational reasons a reduced mass ratio between the ions and electrons of $m_{i} / m_{e}=10^{3}$. The bulk ions are at rest in the simulation box. Their density and temperature are $n_{i}=5 n_{0} / 6$ and $5.5 \mathrm{eV}$. The bulk ions would correspond to the relatively cool upstream plasma ahead of a SNR shock. The ion beam 

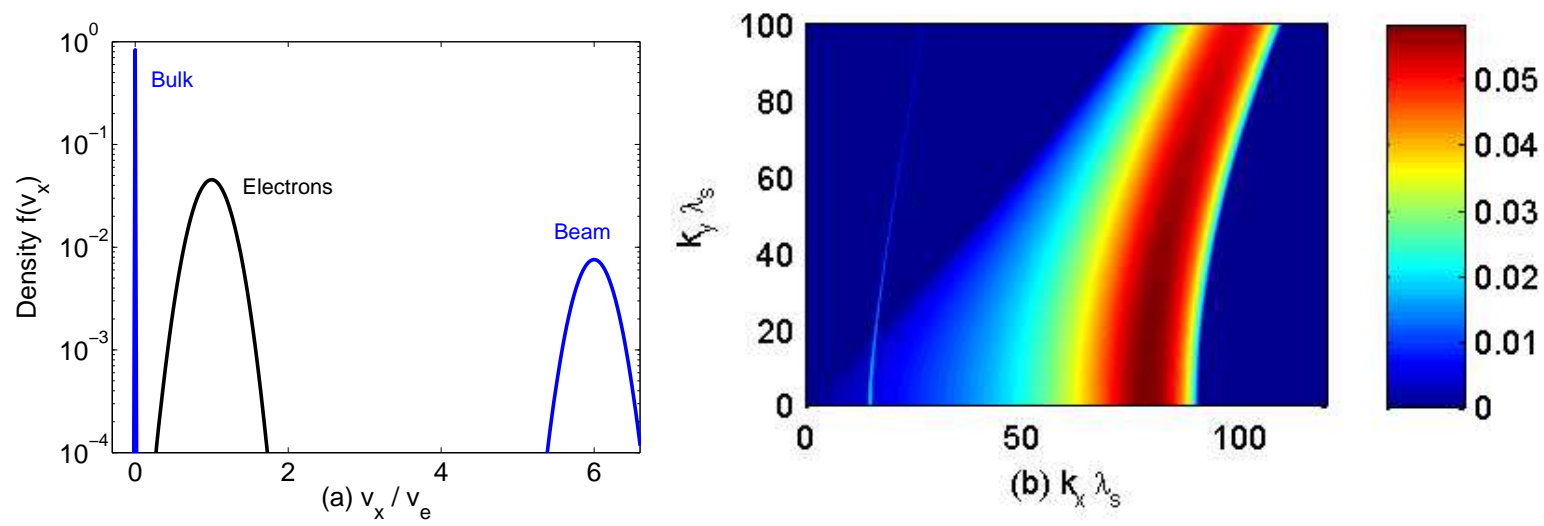

Figure 1. (Color online) The beam distribution is displayed in panel (a). Panel (b) shows the numerical solution of the linear dispersion relation as a function of the beam aligned wave number $k_{x} c / \omega_{p}$ and the perpendicular wave number $k_{y} c / \omega_{p}$. The color corresponds to the exponential growth rate of the wave, expressed in units of the electron plasma frequency $\omega_{p}$. The unstable wave branch at large $k_{x}$ arises from the interaction between background ions and electrons and that at low $k_{x}$ from the interaction between electrons and beam ions.

has the density $n_{b}=n_{0} / 6$ and temperature $10 \mathrm{keV}$. Its mean speed is $v_{b}=6 v_{e}$. The ion beam is much hotter than the bulk ions. Plasma shocks are neither perfectly planar nor elastic reflectors, so the momentum change by the reflection differs for each ion [7], which causes a rise of the temperature. The velocity distribution of the three species is displayed in Fig. 1(a). The plasma is charge neutral by $n_{b}+n_{i}=n_{0}$ and it is initially free of current by $n_{0} v_{e}=n_{b} v_{b}$.

Both BTI's result in electrostatic waves that are practically stationary in the rest frame of the respective ion beam. The real parts of the wave frequencies $\omega_{u 1}, \omega_{u 2}$ and the corresponding wave numbers $k_{u 1}, k_{u 2}$ along $x$ can be estimated in the cold plasma approximation with the dispersion relation $1-\omega_{b}^{2} / \omega^{2}-\omega_{p}^{2} /\left(\omega-k_{x} v_{0}\right)^{2}=0$ valid for one ion beam with plasma frequency $\omega_{b}$ and the electrons with the drift speed $v_{0}$. The bulk ions and the electrons drive waves with the frequency $\omega_{u 1} \ll 1$ and the wave number $k_{u 1} c / \omega_{p} \approx c / v_{e}$ or $k_{u 1} c / \omega_{p} \approx 67$. Since the reference frame of the bulk ions is that of the simulation box, this wave will be practically stationary in the latter.

The instability between the fast ion beam and the electron beam drives waves with $k_{u 2} c / \omega_{p} \approx c /\left(v_{b}-v_{e}\right)$ or $k_{u 2} c / \omega_{p} \approx 13$. Their Doppler shifted frequency is $\omega_{u 2} \approx k_{u 2} v_{b}$ or $\omega_{u 2} \approx 1.2$ in the box frame of reference. The growth rate of a BTI is $\omega_{i} \approx\left(3 \sqrt{3} \omega_{b}^{2} \omega_{p} / 16\right)^{1 / 3}$ [13] and the growth rate ratio between both BTI's is thus $\omega_{i 1} / \omega_{i 2}=\left(n_{i} / n_{b}\right)^{1 / 3}$. The exponential growth rate of the BTI driven by the bulk ions is 1.8 times that of the BTI driven by the fast ion beam.

In the cold plasma limit, this growth rate is independent of the wavenumber $k_{y}$ orthogonal to the beam velocity vector. A more accurate growth rate map is obtained from the solution of the linear dispersion relation that takes into account thermal effects 
and is valid for waves in the simulation plane with $k^{2}=k_{x}^{2}+k_{y}^{2}$,

$$
\left(\omega^{2} \epsilon_{x x}-k_{y}^{2} c^{2}\right)\left(\omega^{2} \epsilon_{y y}-k_{x}^{2} c^{2}\right)-\left(\omega^{2} \epsilon_{y x}+k_{x} k_{y} c^{2}\right)^{2}=0,
$$

and with the elements of the dielectric tensor

$$
\epsilon_{\alpha \beta}(\mathbf{k}, \omega)=\delta_{\alpha \beta}+\sum_{j} \frac{\omega_{p j}^{2}}{\omega^{2}} \int v_{\alpha} \frac{\partial f_{j}^{0}}{\partial v_{\beta}} d^{3} v+\sum_{j} \frac{\omega_{p j}^{2}}{\omega^{2}} \int v_{\alpha} v_{\beta} \frac{\mathbf{k} \cdot\left(\frac{\partial f_{j}^{0}}{\partial \mathbf{v}}\right)}{\omega-\mathbf{k} \cdot \mathbf{v}} d^{3} v
$$

where $\omega_{p j}$ and $f_{j}^{0}$ are the plasma frequency and equilibrium distribution function of the $j^{\text {th }}$ species, respectively (See section 2 in [41]). We approximate $f_{j}^{0}$ by waterbag distributions with the same densities and drift velocities as the plasma species we introduce in our simulation. The thermal width of each waterbag distribution equals the thermal speed of the corresponding Maxwellian distribution. The waterbag distribution is equivalent to a warm fluid model, and a good approximation to a Maxwellian, if the beam speed is large compared to the thermal spreads [42]. The solution of the linear dispersion relation is shown in Fig. 1(b), which displays the imaginary part of the wave frequency (growth rate) as a function of the beam-aligned wave number $k_{x}$ and of the perpendicular wave number $k_{y}$. The colour corresponds to the growth rate, expressed in units of the electron plasma frequency $\omega_{p}$. The most unstable wave numbers in this growth rate map match the ones from the cold plasma approximation for $k_{y} \approx 0$. Larger values of $k_{y}$ result in increasing values of $k_{x}$ and in lower growth rates.

\subsection{The simulation code and the diagnostics}

Our 2D3V electromagnetic and relativistic PIC simulation code [43] resolves the $x, y$ plane and the particle positions in this plane. It updates all three components of the magnetic field $\mathbf{B}$, of the electric field $\mathbf{E}$, of the current $\mathbf{J}$ and of the relativistic particle momentum p. The code preserves $\nabla \cdot \mathbf{E}=\rho / \epsilon_{0}$ and $\nabla \cdot \mathbf{B}=0$ to round-off precision and evolves the electromagnetic fields in time through

$$
\nabla \times \mathbf{E}=-\frac{\partial \mathbf{B}}{\partial t}, \nabla \times \mathbf{B}=\mu_{0} \epsilon_{0} \frac{\partial \mathbf{E}}{\partial t}+\mu_{0} \mathbf{J} .
$$

An ensemble of computational particles $(\mathrm{CPs})$ is followed in time. Each $\mathrm{CP}$ with the index $i$ of the species $j$ in the simulation has the mass and charge $m_{j}$ and $q_{j}$, the momentum $\mathbf{p}_{i}=m_{j} \Gamma_{i} \mathbf{v}_{i}$ and the position $\mathbf{x}_{i}=\left(x_{i}, y_{i}\right)$. Each CP corresponds to a volume element of the phase space distribution of the particle species it represents and the ratio $q_{j} / m_{j}$ must be equal to that of the corresponding physical particle. The position and momentum of each $\mathrm{CP}$ are updated according to

$$
\frac{d \mathbf{x}_{i}}{d t}=\mathbf{v}_{i}, \frac{d \mathbf{p}_{i}}{d t}=q_{j}\left(\mathbf{E}\left[\mathbf{x}_{i}\right]+\mathbf{v}_{i} \times \mathbf{B}\left[\mathbf{x}_{i}\right]\right) .
$$

The simulation box has the size $L_{x} \times L_{y}=3 c / \omega_{p} \times 4 c / \omega_{p}$, which is resolved by a grid with $N_{x} \times N_{y}=580 \times 760$ quadratic cells with the sidelength $\Delta_{x} \approx 1.2 \lambda_{D}$, where $\lambda_{D}$ is the electron Debye length. The boundary conditions are periodic, which limits the wavenumber spectrum to $k_{x} c / \omega_{p}=2 \pi a / 3$ and $k_{y} c / \omega_{p}=2 \pi b / 4$ with the integers 
$1 \leq a \leq N_{x} / 2$ and $1 \leq b \leq N_{y} / 2$. The short unstable wave has $k_{u 1} L_{x} \approx 200$ and about 32 wave lengths are resolved. The long unstable wave has $k_{u 2} L_{x} \approx 39$ and about 6 wave periods are resolved. Each of the plasma species is represented by $320 \mathrm{CPs}$ per cell. The simulation time $T_{M} \omega_{p}=1200$ is resolved by the time steps $\Delta_{t} \omega_{p}=2.5 \times 10^{-3}$.

We compute the following quantities to monitor the plasma evolution. The electric field energies $D_{e x}(t)=\left(2 \epsilon_{0}\right)^{-1} \int E_{x}^{2}(x, y, t) d x d y$ and $D_{e y}(t)=\left(2 \epsilon_{0}\right)^{-1} \int E_{y}^{2}(x, y, t) d x d y$ show the time-evolution of the BTI's. The energy $D_{b z}(t)=\left(2 \mu_{0}\right)^{-1} \int B_{z}^{2}(x, y, t) d x d y$ reveals if an electron thermal anisotropy $A \neq 0$ with $A=\left\langle v_{x}^{2}\right\rangle /\left\langle v_{y}^{2}\right\rangle-1,\left\langle v_{x}^{2}\right\rangle=$ $\sum_{s}\left(v_{x, s}-v_{e}\right)^{2}$ and $\left\langle v_{y}^{2}\right\rangle=\sum_{s} v_{y, s}^{2}$ results in a magnetic field growth. The summation is here over all computational electrons. The electron mean speed along $\mathrm{x}$ and $\mathrm{y}$ varies in the simulation only by about $10^{-2} v_{t e}$ and can thus be taken to be constant. The kinetic energies of the electrons and of the bulk ions are computed in our relativistic code as $K_{e}(t)=m_{E} c^{2} \sum_{s}\left(\Gamma_{s}-1\right)$ and $K_{i}(t)=m_{I} c^{2} \sum_{s}\left(\Gamma_{s}-1\right)$, where $m_{E}$ and $m_{I}$ are the non-relativistic masses of the computational electrons and ions. The sum is over all CPs of the respective species and $\Gamma_{s}$ the Lorentz factor of the $s^{t h} \mathrm{CP}$. All energies are computed in the rest frame of the bulk ions and they are normalized by $K_{e}(0)=E_{K 0}$. The mean electric field in the simulation box is $\left\langle E_{x}\right\rangle=\left(N_{x} N_{y}\right)^{-1} \sum_{i=1}^{N y} \sum_{j=1}^{N x} E_{x}(i, j, t)$, where $E_{x}(i, j, t)$ is the electric field at the grid cell with the indices $i$ and $j$ at the time $t$. A low pass filter removes the oscillations with a frequency exceeding $\omega_{f}=0.8 \omega_{p}$, which reveals the trend of the curve.

In what follows, the velocities $\mathbf{v}$ and positions $\mathbf{x}$ are normalized to $v_{e}$ and to the electron skin depth $\lambda_{s}=c / \omega_{p}$. The time is multiplied with $\omega_{p}$. Frequencies are given in

units of $\omega_{p}$ and the wave numbers are given as $k c / \omega_{p}$. The electric and magnetic fields are expressed as $e \mathbf{E} / \omega_{p} c m_{e}$ and $e \mathbf{B} / \omega_{p} m_{e}$.

\section{Numerical simulation results}

Electrostatic waves are polarized parallel to their wave vector $\mathbf{k}$. Their spectrum contains oblique modes and $E_{x}$ and $E_{y}$ will both grow. Figure 2 shows the time evolution of their energies. An exponential growth of the electric field energy is observed in the time interval $50<t<150$ with $D_{e x} \approx 10 D_{\text {ey }}$. Fitting $\exp \left(2 \omega_{i} t\right)$ to the energy densities $D_{e x}$ and $D_{e y}$ gives us the amplitude's growth rate $\omega_{i} \approx 0.035$. This growth rate is well below the peak $\omega_{i 1} \approx 0.058$ in Fig. 1(b). $D_{e x}$ and $D_{e y}$ are integrated over the entire simulation box, which averages the field energies over all unstable modes and not just over the fastest-growing ones. The saturation of $D_{e x}$ and $D_{e y}$ coincides with the growth of $K_{e}$ and $K_{i}$, which evidences the saturation of the BTI driven by these two species.

Figure 2(b) reveals that $d_{t}\left\langle E_{x}\right\rangle<0$ during $100<t<150$, which implies by Ampère's law a box-averaged $\left(k_{x}=0\right)$ net current $j_{x}>0$ and thus an electron deceleration in the reference frame of the simulation box. The net current is tied to a change of the mean speed of the electrons in response to the BTI between the bulk ions and electrons and their re-distribution in phase space by its saturation. This current is not compensated by a change in the ion mean speed due to the large ion inertia. The 

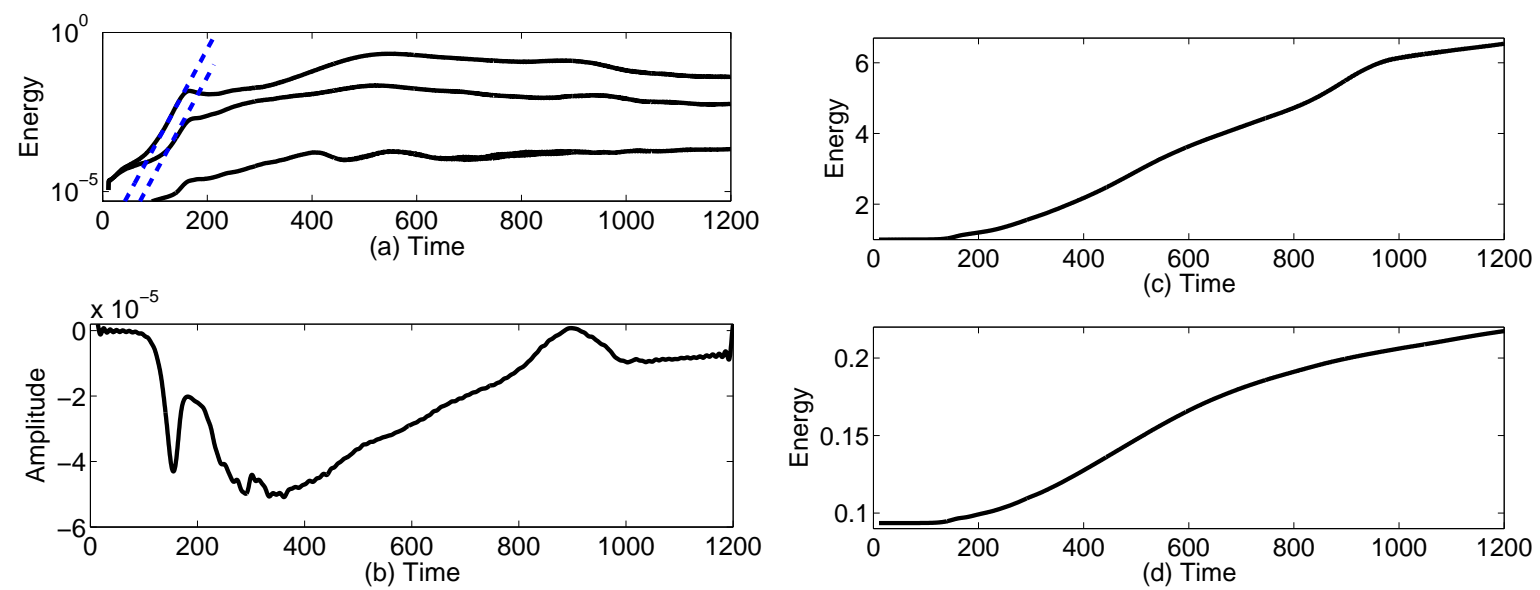

Figure 2. (Colour online) The electric $D_{e x}$ (upper curve), $D_{e y}$ and the magnetic $D_{b z}$ (lower curve) energies are displayed in panel (a). The dashed blue lines are exponential fits with the same growth rate. Panel (b) shows the box-averaged electric field amplitude $\left\langle E_{x}\right\rangle$. The electron energy $K_{e}(t)$ is shown in panel (c) and that of the bulk ions in panel (d). All energies are normalized to $E_{K 0}$.

electrons are accelerated by the $\left\langle E_{x}\right\rangle<0$, so that the electron mean speed along $x$ remains close to $v_{e}$. The required acceleration of electrons by $\left\langle E_{x}\right\rangle$ is small. Even the peak electric field $\left\langle E_{x}\right\rangle \approx-4 \times 10^{-5}$ can change the electron speed by only $\Delta_{v} \approx 3 \times 10^{-3}$ during a time interval $\Delta_{T} \approx 1$. A rapid switch to $d_{t}\left\langle E_{x}\right\rangle>0$ takes place at $t \approx 150$, which coincides with the onset of the growth of the electron and bulk ion energies and is thus related to the nonlinear saturation of the BTI between these species.

All displayed field components and the electron energy continue to grow after $t \approx 150$. $D_{e x}$ and $D_{e y}$ grow by another order of magnitude until $t \approx 500$ when they reach their maximum. We show below that this growth is caused by the BTI between the fast ion beam and the electrons. The growth rates of $D_{e x}$ and $D_{e y}$ are well below that of the initial growth phase prior to $t \approx 150$ and $D_{e x}$ is not proportional to $D_{e y}$. We observe a $d_{t}\left\langle E_{x}\right\rangle>0$ and thus a change of the sign of the net current to positive values after $t \approx 350$. Electrons start to interact with the waves driven by the second BTI and are accelerated to positive $v_{x}$. An exponential growth of $D_{b z}$ between $200<t<400$ is observed and it remains approximately constant after this time. The magnetic energy remains 2-3 orders of magnitude below that of the electric field components, which implies an essentially electrostatic plasma dynamics. The electron energy $K_{e}$, which takes into account their bulk flow, grows practically linearly up to a value $6 E_{K 0}$ in the interval between $150<t<1000$.

\subsection{Saturation of the instability between electrons and bulk ions}

Figure 3 shows the electric fields in a part of the simulation box at $t=150$. The structures in $E_{x}$ and $E_{y}$ belong to waves with wave vectors that are in some cases tilted with respect to the beam velocity vector. Their amplitude is two orders of magnitude 

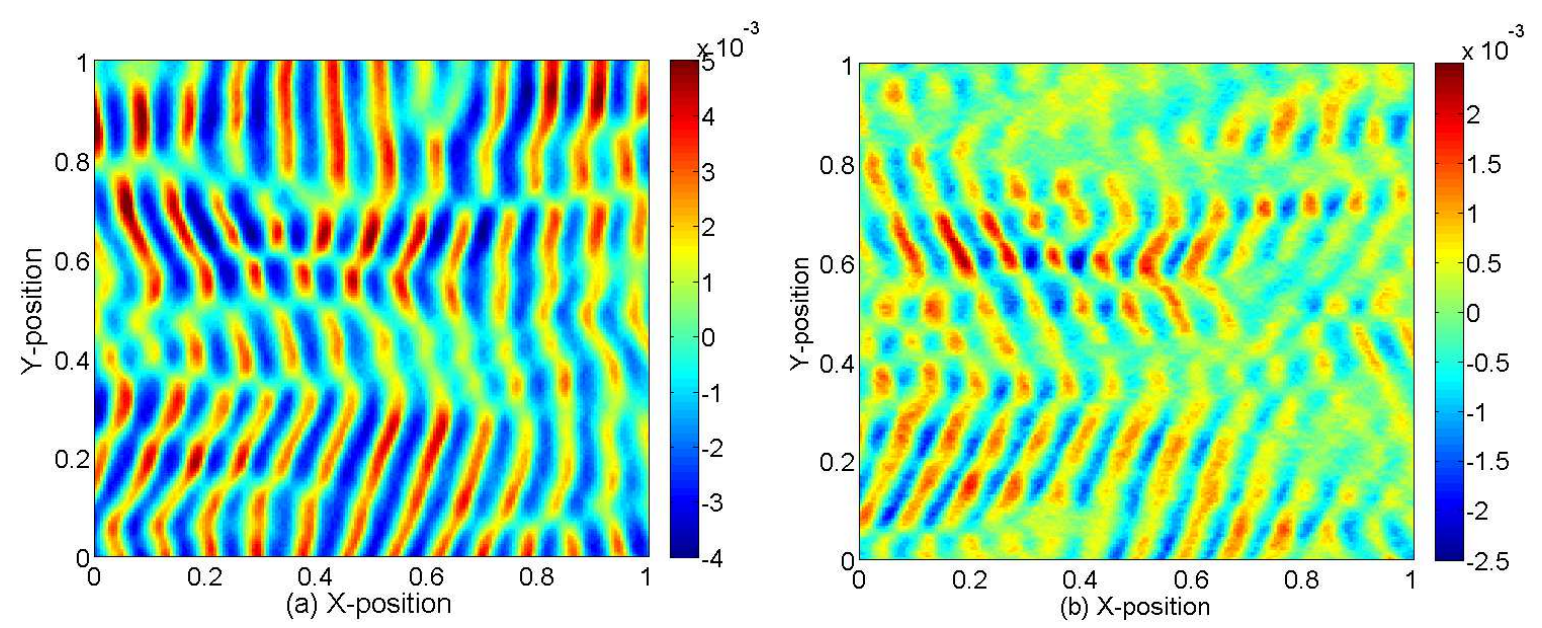

Figure 3. (Color online) The electric field distributions in a subinterval of the simulation box at $t=150$ : $E_{x}$ is shown in panel (a) and $E_{y}$ in panel (b).

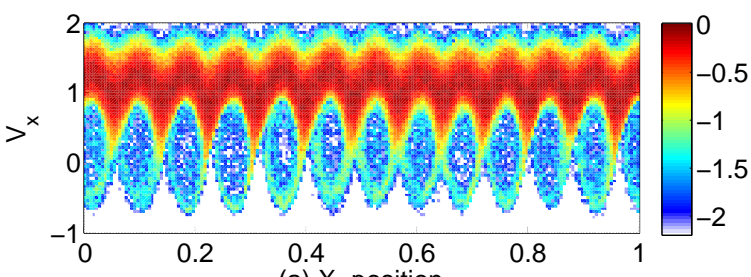

(a) X-position

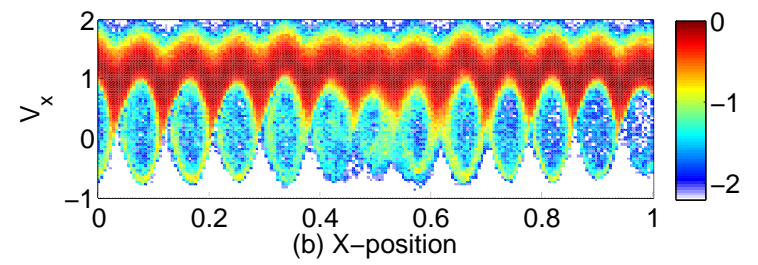

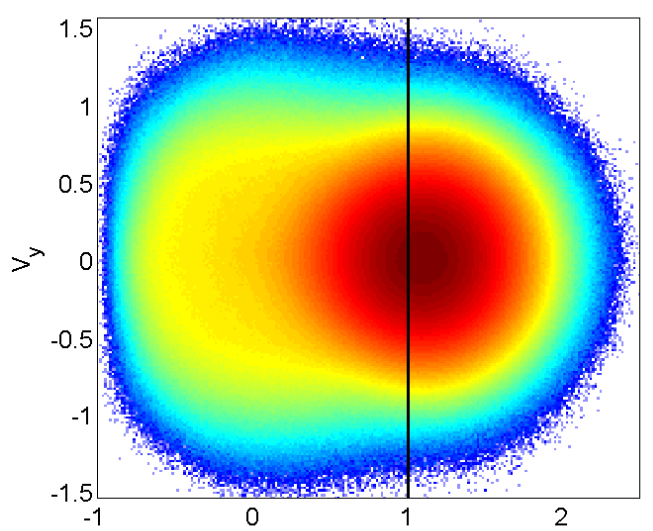

(c) $v_{x}$

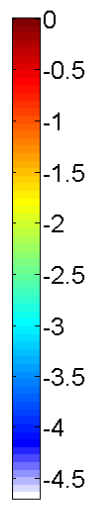

Figure 4. (Color online) Electron phase space distributions at $t=150$ : Panel (a) and (b) show phase space projections onto the $x, v_{x}$ plane. The distribution is integrated over $0<y<0.05$ in (a) and over $1.55<y<1.6$ in (b). The electron distribution has been integrated over all $x, y, v_{z}$ for the projection onto the $v_{x}, v_{y}$ plane in panel (c). The vertical line corresponds to $v_{x}=v_{e}$. All distributions are normalized to their respective maxima and the colour scale is 10-logarithmic.

larger than the mean electric field $\left\langle E_{x}\right\rangle$ in Fig. 2(b). Such wave fields have also been observed previously in electrostatic PIC simulations [18]. The $E_{x}$ and $E_{y}$ are projections of the obliquely oriented electric field onto the $x$ and $y$ axes, explaining why $D_{e x} \propto D_{\text {ey }}$ until $t=150$. The wavelength of the structures is $\lambda \approx 0.06$ or $k=\left(k_{x}^{2}+k_{y}^{2}\right)^{1 / 2} \approx 100$. This value is in line with the wave numbers of the oblique modes in Fig. 1(b).

Various projections of the resolved electron phase space distribution $f\left(x, y, v_{x}, v_{y}, v_{z}\right)$ at $t=150$ are shown in Fig. 4. The phase space projection on the $x, v_{x}$ plane integrates this distribution over all $v_{y}, v_{z}$ and over ten grid cells along $y$. Figures 4 (a) and (b) integrate the distribution over $0<y<0.05$ and over $1.55<y<1.6$, respectively. Figures 

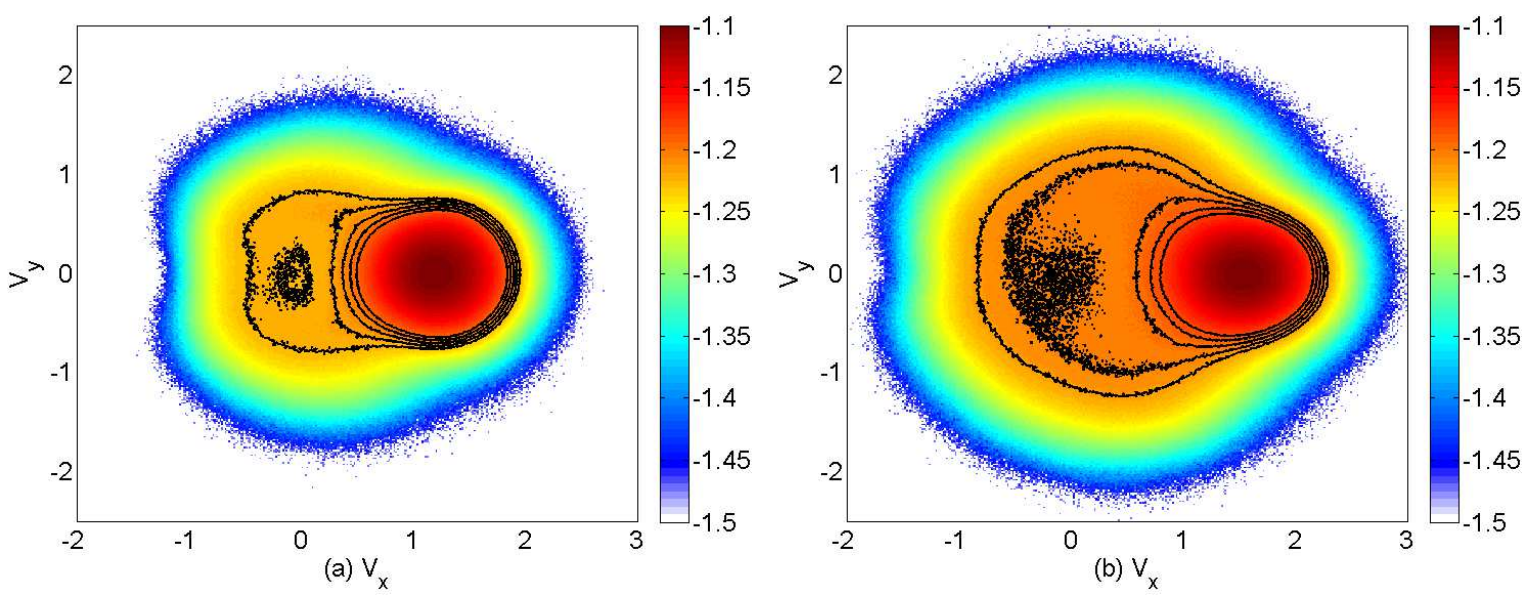

Figure 5. (Color online) Evolution of the electron velocity distribution: Panel (a) shows the electron distribution at $t=200$ and panel (b) shows it at $t=300$. Both distributions are normalized to the peak value at $t=150$ and the colour scale is 10-logarithmic. The black curves are the contour lines $-1.24,-1.23,-1.22,-1.21,-1.20$.

4(a) and (b) reveal electron phase space holes, which arise when electrostatic waves saturate [34]. The spatial width of each phase space hole corresponds to one wave period of the electrostatic waves driven by the BTI. The exponential growth phase of $D_{e x}$ and $D_{e y}$ thus ends at $t=150$ as usual due to the trapping of electrons. The electrons are trapped by a potential, which is practically stationary in the rest frame of the bulk ions. This is evidenced by the supplementary movie 1, which shows a time animation of Fig. 4(a). The electrons gyrate around $v_{x}=0$ at early times. The mean speed of the trapped electrons inside of a phase space hole is less than $v_{e}$, which can be seen in Fig. 4. A significant fraction of the electrons has been slowed down at $t=150$, which reduces the modulus of the electron current. The electron current is negative because $v_{e}>0$. The ions hardly react to the electric field because $m_{i} \gg m_{e}$ and they can not compensate the change of the electron current. We obtain a macroscopic box-averaged $\left\langle j_{x}\right\rangle>0$, which explains why $d_{t}\left\langle E_{x}\right\rangle<0$ in Fig. 2(b). The mean electric field $\left\langle E_{x}\right\rangle<0$ in Fig. 2(b) accelerates all electrons in the positive direction, which will reduce $\left\langle j_{x}\right\rangle$. The maximum of the phase space density in Fig. 4(c) is thus found at $v_{x}>1$. The electrons in this core population have not been trapped as we can see from Figs $4(a, b)$ and from movie 1, but they have all gained speed along $x$. The trapped electrons in Fig. 4 (c) are found at $v_{x}<1$. The electron distribution is stretched out along $v_{y}$, because the electrons are trapped by oblique waves (See Fig. [3).

The electrons are accelerated by $\left\langle E_{x}\right\rangle<0$ (Fig. 2(b)) into the positive x-direction and they are heated by their interaction with the localized electric field structures (Fig. 3). Figure 5 demonstrates this by a comparison of the electron velocity distributions at the times $t=200$ and $t=300$, when the modulus of $\left\langle E_{x}\right\rangle$ is largest. The velocity that corresponds to the maximum of the electron phase space density is found at $v_{x} \approx 1.2$ at $t=200$ and at $v_{x} \approx 1.6$ at $t=300$. The distribution widens in velocity space, which corresponds to an increasing thermal speed, and it adopts an increasingly circular shape. 

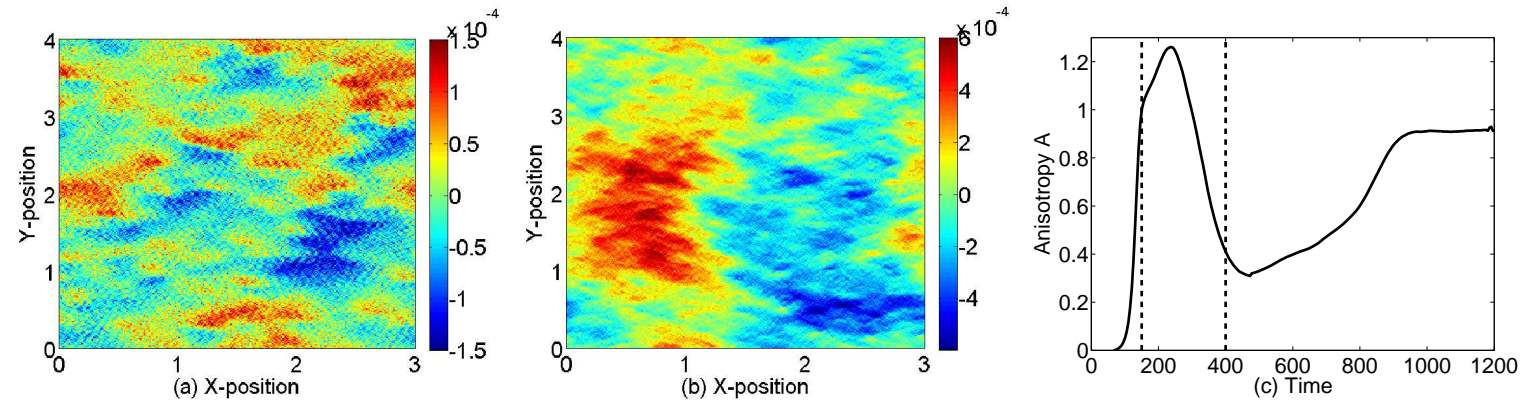

Figure 6. (Color online) The $B_{z}$ component of the magnetic field at the times $t=150$ (a) and $t=400$ (b). The full simulation grid is displayed. The thermal anisotropy $A$ is shown as a function of time $\omega_{p} t$ in (c) and the times $t=150$ and $t=400$ are overplotted as vertical dashed lines.

The centre of the hot circular distribution is at $v_{x} \approx 0.3$ at $t=300$. We can not observe a density distribution in Fig. 5 that resembles the crescent found in Ref. [18].

The electron distribution in Fig. 44(c) can be subdivided into a thermal core population centred at $v_{x} \approx 1.2$ and $v_{y} \approx 0$ with a high density and a thermally anisotropic hot electron population with a lower density. The low-density electron population is elongated along $v_{x}$ in Fig. 5(a) and it has become almost circular at $t=300$ in Fig. 5(b). It is thus fair to assume that this thermally anisotropic electron population is responsible for the growth of $D_{b z}$ in Fig. 2(a) and that the magnetic field growth ceases once the electron distribution is isotropic in velocity space. A thermally anisotropic electron distribution drives waves with a wave vector, which is aligned with the direction along which the electrons are cool [21]. We thus expect a wave vector of the magnetowaves that is almost parallel to the $y$-direction. The magnetic instability is driven by currents in the $\mathrm{x}-\mathrm{y}$ plane and the unstable magnetic field component is thus aligned with the z-direction. Indeed only $D_{b z}$ grows in our simulation, while the other magnetic field components remain at noise levels (not shown).

Figure [6 shows the electron thermal anisotropy $A(t)$ and compares the spatial distribution of $B_{z}$ at the time $t=150$, which is just before the exponential growth phase of $D_{b z}$ in Fig. 2(a), and at $t=400$, when the exponential growth phase of $D_{b z}$ ends. The magnetic field is initially weak and the structures are small. The saturation of the BTI between the bulk plasma and the electrons has resulted in a rapid growth of $A$, which has reached $A \approx 1$ at $t=150$. This value corresponds to an electron thermal energy along $x$ that is twice that along $y$. The anisotropy reaches its peak at $t \approx 250$ and decreases thereafter to a value $A \approx 0.4$ at $t=400$, when a large coherent magnetic field structure has emerged in Fig. 6(b). The size of this patch is of the order of an electron skin depth in both spatial directions and the magnetic amplitude gives a ratio between the electron cyclotron frequency and the plasma frequency of $6 \times 10^{-4}$, which is comparable to the ratio found in the interstellar medium [32]. The field patch is not thermal noise. Thermal noise [44] is linked to charge and current density fluctuations due to the finite number of computational particles and its coherence length is comparable 
to the grid cell size. The structure centred at $x=0.7$ and $y=1.7$ in 6 (b) is, however, not the plane wave with a wave vector parallel to the y-axis, which we would expect from a TAWI [24]. A magnetic structure is growing while $A$ is large, but we can not attribute it to an elementary instability such as a TAWI.

\subsection{Saturation of the instability between electrons and the fast ion beam}

The comparison of Figs. 1(a) and [5) demonstrates that the mean electric field $\left\langle E_{x}\right\rangle$ in Fig. 2(b) has accelerated the bulk of the electrons from $v_{x}=1$ to $v_{x} \approx 1.7$. The velocity gap $\Delta v_{x}$ between the value $v_{x}$, where the electron phase space density reaches its maximum, and the mean speed $v_{b}=6$ of the fast beam has thus decreased from 5 to 4.3. The change of $\Delta v_{x}$ should result in a larger wave number of the unstable electrostatic waves than the now normalized $k_{u 2}=1 /\left(v_{b}-v_{e}\right) \approx 13$ estimated in section 2. This relative speed $\Delta v_{x}$ expressed in units of the electron thermal speeds is also well below the value used in Ref. [18], in particular if we take into account that the electrons have been heated up by the saturation of the first BTI.

Figures $7(a, b)$ reveal that this second BTI has already commenced to grow at $t=400$. Movie 1 shows a wave perturbation after $t \approx 300$ of the electron distribution that propagates at a high speed to increasing $x$, which corresponds to the wave driven by the BTI between the electrons and the fast beam. Strong modulations of the electron distribution with $1.5<v_{x}<3$ are present. Their wave number is $k_{o}=14 \pi / 3 \approx 14.6$, which is indeed larger than $k_{u 2}$. However, $k_{o}$ may not correspond to the true fastest growing wave because the periodic boundary conditions enforce a discrete wave spectrum. The electron velocity distribution in Fig. 7(c) reveals that the dilute electron component forms an almost circular distribution centred at $v_{x} \approx 0.5$ and $v_{y} \approx 0$. The contour line corresponding to $10^{-1.18}$ at $v_{x} \approx 1$ is almost aligned with the $v_{y}$-axis. It resembles the crescent in Ref. [18, but it is much less pronounced.

Figure 8 shows the electric field distribution at $t=500$, when $D_{e x}$ and $D_{e y}$ reach their peak values in Fig. 2(a). The structures in $E_{x}$ and $E_{y}$ show a spatial correlation due to the obliquity of the wave front. The characteristic tilt angle between the wave vector and the x-axis is less than the one in Fig. 2 and thus $\left|E_{y}\right| \ll\left|E_{x}\right|$. Internal filamentary structures are visible in the intervals with $\left|E_{x}\right| \gg 0$. The filamentary structures are relatively stronger in $E_{y}$ compared to $E_{x}$, which may explain why $D_{e x}$ does no longer grow in unison with $D_{e y}$ in Fig. 2(a) at this time. The electric field amplitude in Fig. 8(a) exceeds that in Fig. 3(a) by a factor of 4 and the wave length is larger by a factor 5. The electrostatic potential, which is driven by the BTI between the electrons and the fast ion beam, thus exceeds that of the faster growing BTI by a factor of $\approx 20$ and should give trapped electron islands larger than those in Fig. 4.

Some electrons are trapped at the time $t=500$ by this faster wave, which is the time when $D_{e x}$ saturates in Fig. 2(a). The supplementary movie 2, which shows the spatially integrated phase space distribution as a function of $v_{x}, v_{y}$ and corresponds to Fig. 9(c), demonstrates that the number of trapped electrons rapidly increases after this 


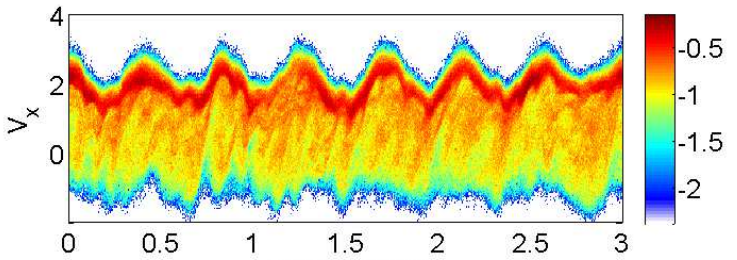

(a) X-position

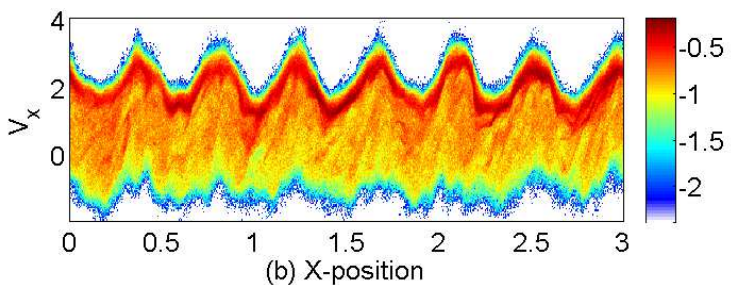

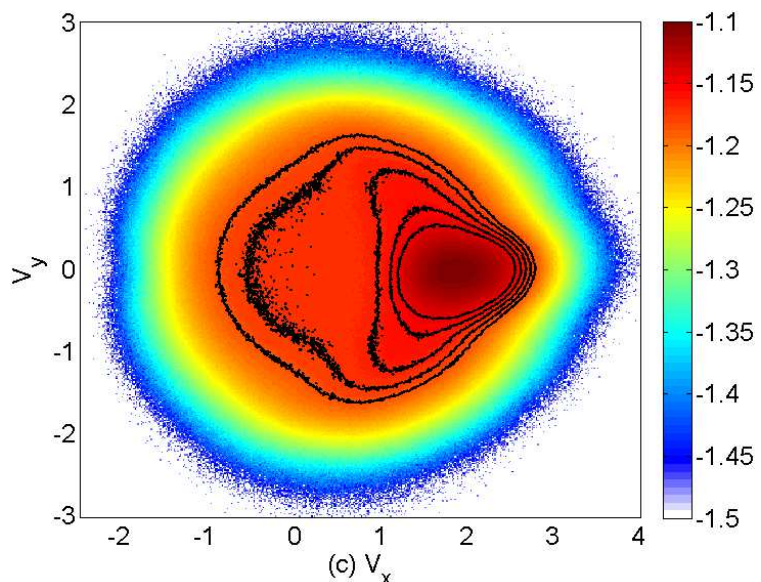

(c) $V_{x}$

Figure 7. (Color online) Electron phase space distributions at $t=400$ : Panel (a) and (b) show phase space projections onto the $x, v_{x}$ plane. The distribution is integrated over $0<y<0.05$ in (a) and over $1.55<y<1.6$ in (b). The electron distribution has been integrated over all $x, y, v_{z}$ for the projection onto the $v_{x}, v_{y}$ plane in panel (c). All distributions are normalized to their respective maxima at $t=150$ and the colour scale is 10-logarithmic. The black curves in (c) are the contour lines -1.2, -1.19, -1.18, $-1.17,-1.16$.
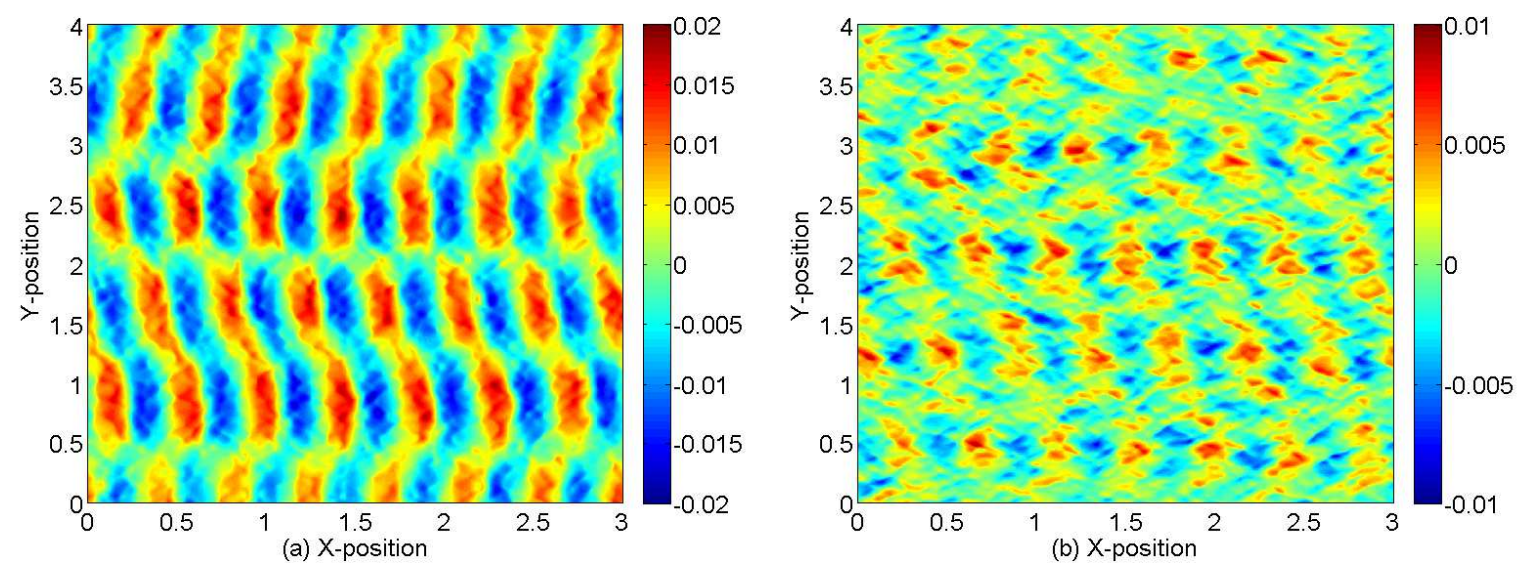

Figure 8. (Color online) The electric field distributions at $t=500: E_{x}$ is shown in panel (a) and $E_{y}$ in panel (b).

time. The black lines correspond to the speeds of both ion beams. Figures $9(a, b)$ show that the electron velocity distribution is not sinusoidal, which is a sign of a nonlinear wave, and that some electrons have speeds of $v_{x}>5$. These electrons are found close to the cusps of the electron distribution with $v_{x} \approx 5$, which correspond to the unstable equilibrium point of a periodic electrostatic potential that moves with a speed $\approx v_{b}$. The fastest electrons have just started their periodic motion in the electrostatic potential of the wave fronts with a wave vector, which is parallel to the $\mathrm{x}$-axis.

The electron velocity distribution in Fig. 9(c) reveals that a small fraction of electrons have been accelerated up to $v_{x} \approx 10$. These electrons have reached the stable 

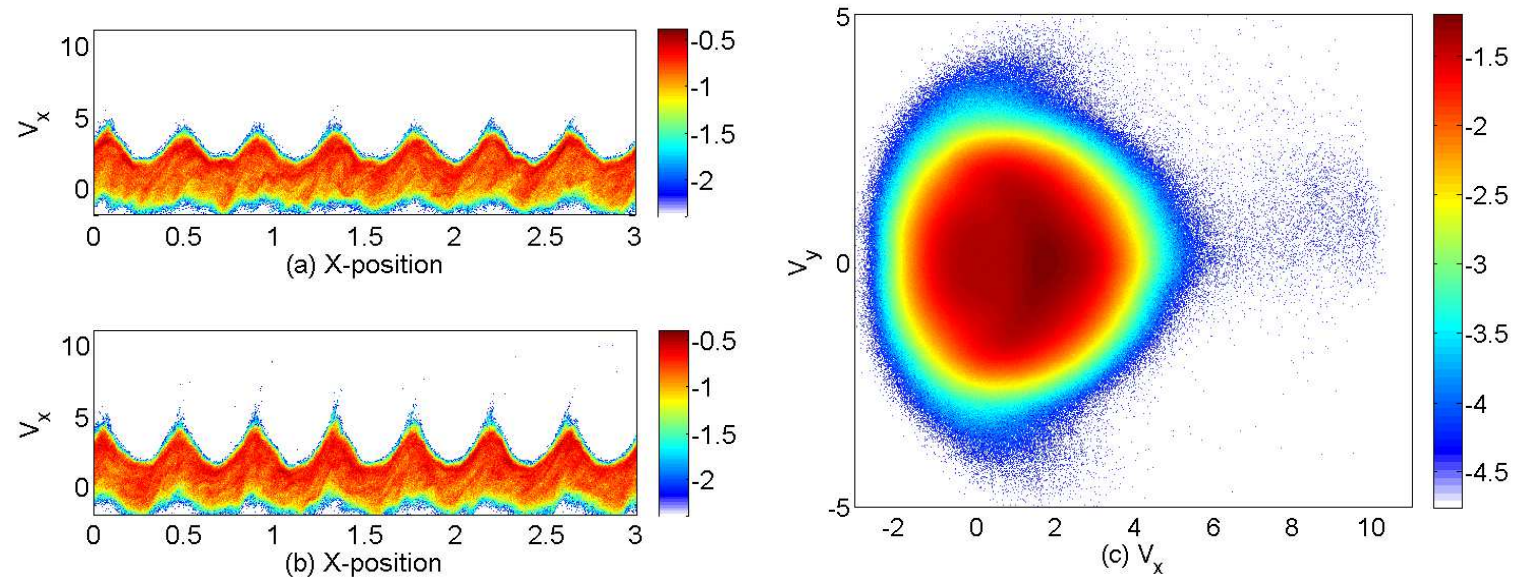

Figure 9. (Color online) Electron phase space distributions at $t=500$ : Panel (a) and (b) show phase space projections onto the $x, v_{x}$ plane. The distribution is integrated over $0<y<0.05$ in (a) and over $1.55<y<1.6$ in (b). The electron distribution has been integrated over all $x, y, v_{z}$ for the projection onto the $v_{x}, v_{y}$ plane in panel (c). All distributions are normalized to their respective maxima at $t=150$ and the colour scale is 10-logarithmic.

equilibrium point of the electrostatic potential and their kinetic energy reaches its peak value. The electron distribution extends here and at later times (Movie 2) over a velocity interval that does not exceed $20 v_{e}$ or $0.3 \mathrm{c}$ and the electrons thus remain nonrelativistic. The velocity distribution reveals non-thermal electrons at $v_{x} \approx 1$ and at $\left|v_{y}\right| \approx 4$. These electrons must have gained speed by their interaction with the oblique modes. They can interact more easily with electrons than the beam aligned mode, because their phase speed is lower [18]. The interaction of electrons with the oblique modes results in a velocity distribution, which resembles a crescent. This saturation mechanism is dominant, if the thermal spread of the electrons is small compared to the beam speed. The beam speed was 10 times the electron thermal speed in Ref. [18]. If the electron thermal spread is comparable to the beam speed, like for the instability between the bulk ions and the electrons, then the beam aligned modes can easily trap the electrons in the high-energy tail of the electron distribution and no crescent develops. The larger gap between the speed $v_{x} \approx 3.5$ of the fastest electrons in Fig. 7(c) and the ion beam speed $v_{b}=6$ apparently puts the electron interaction with oblique modes and with beamaligned modes on an equal footing. Movie 2 clearly shows that electrons are accelerated along $v_{x}$ at large $v_{x}$ and along $v_{y}$ for $v_{x} \approx 0$ after $t \approx 450$.

The interaction of the electrons with the electrostatic potentials of the long waves scatters them. Scattering the electrons results in dissipation, which affects the current and charge density distribution of the plasma. Figure 10 shows the distributions of $E_{x}$ and $B_{z}$ at $T_{M}=1200$. Strong electrostatic waves are still present. The magnetic field reveals strong noise fluctuations on a Debye-length scale, which we attribute to thermal noise. The typical amplitudes of $B_{z}$ are large; the fluctuation amplitude increases with 

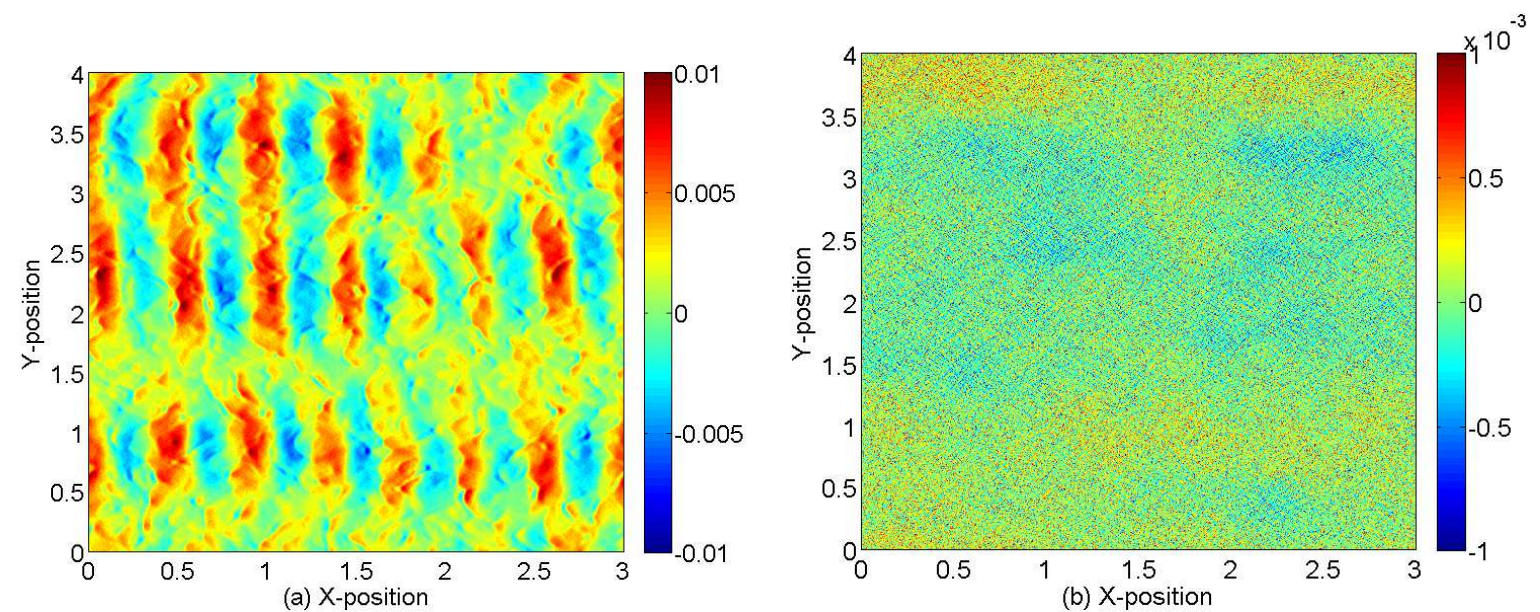

Figure 10. (Color online) The electric field distributions at $t=1200: E_{x}$ is shown in panel (a) and $B_{z}$ in panel (b).
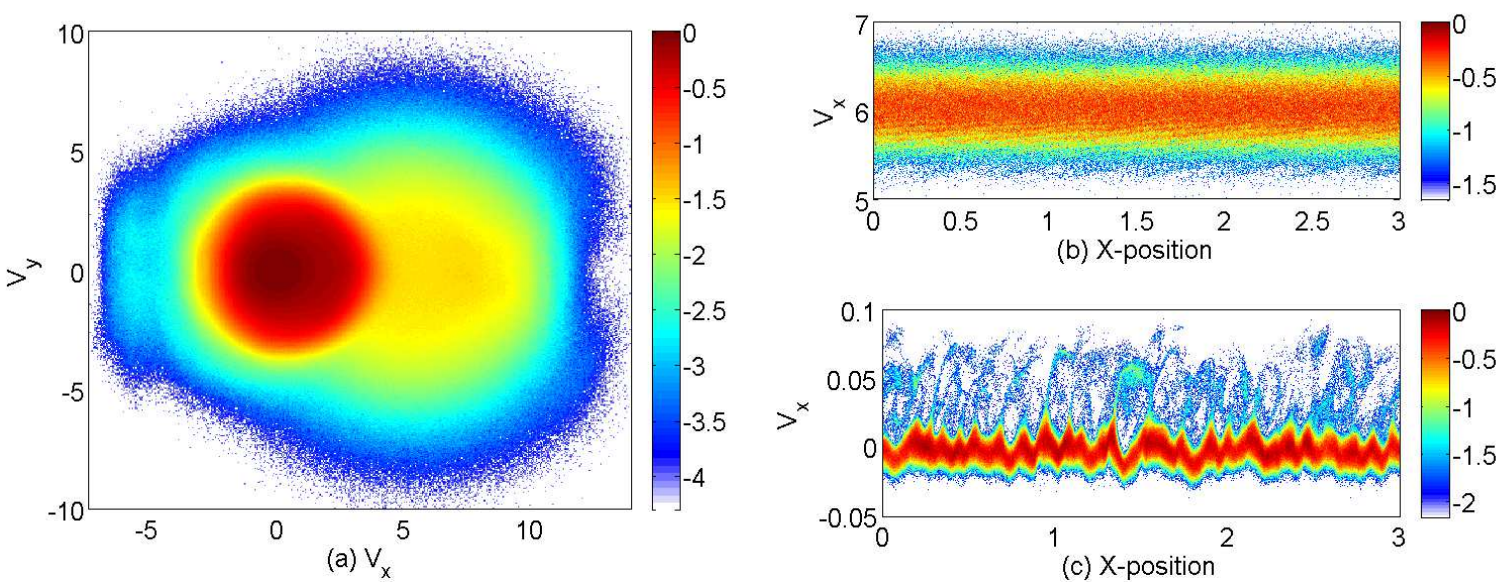

Figure 11. (Color online) Particle distribution functions at $t=1200$ : Panel (a) shows the electron velocity distribution. Panel (b) and (c) show the ion distributions integrated over the interval $0<y<0.05$. All distributions are normalized to their peak value at $t=1200$ and the colour scale is 10 -logarithmic.

the electron temperature. We conclude from Fig. 10(b) that the TAWI, even if it has been responsible for the magnetic field growth in Fig. 6, is not an efficient source of coherent magnetic fields for the selected parameters.

The reason for the discrepancy between the lifetimes of the electrostatic and magnetic structures becomes evident from the plasma distribution functions shown in Fig. 11. The electron velocity distribution in Fig. 11(a) has its peak density at $v_{x} \approx 0$ and $v_{y} \approx 0$. The electrons accumulate at speeds, which correspond to the phase speed of the waves driven by the BTI between bulk ions and electrons. An approximately circular electron velocity distribution extends up to $\sqrt{v_{x}^{2}+v_{y}^{2}} \approx 3$. The electron scattering is isotropic in the rest frame of the slow waves. A second circular structure, albeit with a lower number density, is observed at $v_{x} \approx 6$. These electrons accumulate at the 
phase velocity of the waves driven by the BTI between electrons and the fast ion beam. The electron scattering is apparently isotropic in the rest frame of the fast waves. The electrons are thus scattered by two systems of electrostatic waves that have a different wave length and that move at different speeds.

The scattering should result in the rapid thermalisation of the electrons and in a dissipation of large-scale electron currents in the simulation plane. This dissipation explains why the $B_{z}$ field in Fig. 10(b) is no longer coherent over large spatial scales. The electrostatic fields can also be supported by modulations of the ion velocity and density and they are thus robust against the electron scattering. The beam ions in Fig. 11(b) do not show any non-thermal features and the density modulation is in the linear regime. Their high temperature implies that the beam ions can support the large electrostatic fields in Fig. 10(a) without developing nonlinear velocity oscillations. The bulk ions in Fig. 11(c) show strong velocity oscillations. Their low thermal pressure implies that they can not sustain large electrostatic fields without showing non-thermal signatures, like accelerated ions.

\section{Discussion}

We have examined the growth and saturation of electrostatic Buneman-type instabilities (BTI's) with the help of a particle-in-cell simulation. Our system of two counterstreaming ion beams is representative for the foreshock region of supernova remnant shocks. The bulk ions and the electrons are the upstream plasma, while the ion beam corresponds to the shock-reflected ions. The system has initially been charge and current neutral and the plasma has been field-free, which is idealized. Electrostatic unmagnetized shocks have been observed experimentally and numerically [45, 46], but their speed is typically well below the electron thermal speed. Their shock-reflected ion beam can thus not be faster than the electron thermal speed. We assume here that the SNR shock carries a magnetic field perpendicular to the shock normal, which reflects the incoming upstream ions even at high shock speeds, that is weak enough to be negligible with regard to the growth of the BTI. The latter implies that, in the electron rest frame, the electron cyclotron frequency should be well below the frequency of the waves driven by the BTI. A stronger magnetic field yields additional unstable wave branches [16] and isotropizes the electrons more rapidly orthogonally to the magnetic field.

The simulation has shown that two wave modes are growing. One instability branch corresponds to the BTI between the bulk ions and the electrons. It is the faster growing one. The second branch develops between the fast ion beam and the electrons. The large phase speed of its waves can accelerate electrons to larger speeds and this instability is thus more powerful. The electron speeds do, however, remain nonrelativistic as in previous 1D simulation studies [13]. The BTI in unmagnetized plasma can thus not account for electron injection into the diffusive acceleration and magnetic fields would be necessary for this purpose [47]. Both wave branches support a continuos wave spectrum ranging from the beam-aligned modes with a high phase speed to the slower oblique 
modes [18]. The low relative speed between the electrons and the bulk ions implied that the instability saturated by the conventional trapping of electrons by beam-aligned modes [34]. The growth of magnetic fields could be observed after this saturation. Their energy density was, however, too low to identify unambigously the instability responsible for their growth. A likely candidate is the TAWI [31]. The peak ratio between the electron cyclotron frequency and the plasma frequency remained below $10^{-3}$, which is comparable to the equivalent in the ISM.

The electrostatic instability between the fast ion beam and the heated electrons developed at a later time. It saturated by the trapping of electrons by beam-aligned modes and by obliquely propagating slower modes. The phase space distribution revealed the simultaneous development of phase space holes and of the crescent distribution, which was reported first in Ref. [18]. The second mechanism becomes dominant, if the beam speed exceeds by far the electron thermal spread, which was not the case here. The formation of such a distribution might also be aggravated by the much hotter ions we have used here. No growth of a strong coherent magnetic field could be observed, in spite of a stable thermal anisotropy of the electron distribution.

It is unclear, why the TAWI is inefficient here. A possible reason is that the magnetic interaction between electrons, which gives rise to the TAWI, competes with the electron interaction with the electrostatic wave fields. The latter has not been taken into account in numerical studies and may inhibit the formation of stable current filaments that are needed to sustain strong magnetic fields. We conclude that, at least for our simulation parameters, the TAWI driven by the BTI can not magnetize SNR shocks due to its short life time and low peak amplitude of the magnetic fields.

Acknowledgements: This work was supported by Vetenskapsrådet (DNR 20104063), by EPSRC (EP/D043808/1, EP/D06337X/1, EP/I031766/1), by Consejeria de Educacion y Ciencia (ENE2009-09276), by the Leverhulme Trust (ECF-2011-383) and by the Junta de Comunidades de Castilla-La Mancha (PAI08-0182-3162). Computer time and support was provided by the HPC2N in Umeå.

[1] J P Eastwood, E A Lucek, C Mazelle, K Meziane, Y Narita, J Pickett, R A Treumann 2005 Space Sci. Rev. 11841

[2] P J Cargill and K Papadopoulos 1988 Astrophys. J. 329 L29

[3] K G McClements, R O Dendy, R Bingham, J G Kirk and L O Drury 1997 Mon. Not. R. Astron. Soc. 291241

[4] H Schmitz, S C Chapman and R O Dendy 2002 Astrophys. J. 570637

[5] S C Chapman, R E Lee and R O Dendy 2005 Space Sci. Rev. 1215

[6] T Umeda, Y Masahiro and R Yamazaki 2008 Astrophys. J. 681 L85

[7] S Matsukiyo and M Scholer 2006 J. Geophys. Res. 111 A06104

[8] B Lembege, P Savoini, P Hellinger and P M Travnicek 2009 J. Geophys. Res. 114 A03217

[9] A M Bykov and R A Treumann 2011 Astron. Astrophys. Rev. 1942

[10] O Buneman 1958 Phys. Rev. Lett. 18

[11] O Buneman 1959 Phys. Rev. 115503

[12] J M Dawson 1983 Rev. Mod. Phys. 55403

[13] M E Dieckmann, P Ljung, A Ynnerman and K G McClements 2000 Phys. Plasmas 75171

[14] N Shimada and M Hoshino 2003 Phys. Plasmas 101113

[15] J Pavan, P H Yoon and T Umeda 2011 Phys. Plasmas 18042307 
[16] M E Dieckmann, K G McClements, S C Chapman, R O Dendy and L O C Drury 2000 Astron. Astrophys. 356377

[17] N Shimada and M Hoshino 2004 Phys. Plasmas 111840

[18] T Amano and M Hoshino 2009 Phys. Plasmas 16102901

[19] Y Ohira and F Takahara 2007 Astrophys. J. 661 L171

[20] M E Dieckmann and A Bret 2008 New J. Phys. 10013029

[21] E S Weibel 1959 Phys. Rev. Lett. 5283

[22] A Bret 2007 Contrib. Plasma Phys. 47113

[23] H H Kaang, C M Ryu and P H Yoon 2009 Phys. Plasmas 16082103

[24] A Stockem, M E Dieckmann and R Schlickeiser 2009 Plasma Phys. Controll. Fusion 51075014

[25] L Palodhi, F Califano and F Pegoraro 2009 Plasma Phys. Controll. Fusion 51125006

[26] A Stockem, M E Dieckmann and R Schlickeiser 2010 Plasma Phys. Controll. Fusion 52, 085009

[27] M E Innocenti, M Lazar, S Markidis, G Lapenta and S Poedts 2011 Phys. Plasmas 18052104

[28] R C Tautz 2011 Phys. Plasmas 18012101

[29] D V Romanov, V Y Bychenkov, W Rozmus, C E Capjack and R Fedosejevs 2004 Phys. Rev. Lett. 93215004

[30] J Niemiec, M Pohl, T Stroman and K Nishikawa 2008 Astrophys. J. 6841174

[31] R Schlickeiser 2005 Plasma Phys. Controll. Fusion 47 A205

[32] EG Berezhko, LT Ksenofontov and HJ Volk 2003 Astron. Astrophys. 412 L11

[33] A R Bell 2004 Mon. Not. R. Astron. Soc. 353550

[34] K V Roberts and H L Berk 1967 Phys. Rev. Lett. 19297

[35] D L Newman, M V Goldman, M Spector and F Perez 2001 Phys. Rev. Lett. 861239

[36] C Lancelotti and J J Dorning 2003 Phys. Rev. E 68026406

[37] A Luque and H Schamel 2005 Phys. Rep. 415261

[38] G Sarri et al 2010 Phys. Plasmas 17010701

[39] MY Wu, QM Lu, C Huang and S Wang 2010 J. Geophys. Res. 115 A10245

[40] D Prono, B Ecker, N Bergstrom and J Benford 1975 Phys. Rev. Lett. 35438

[41] A Bret, L. Gremillet and M. E. Dieckmann 2010 Phys. Plasmas 17120501

[42] A Bret and C Deutsch 2006 Phys. Plasmas 13042106

[43] J W Eastwood 1991 Comput. Phys. Commun. 64252

[44] ME Dieckmann, A Ynnerman, SC Chapman, G Rowlands and N Andersson 2004 Phys. Scripta 69456

[45] L Romagnani et al 2008 Phys. Rev. Lett. 101025004

[46] G Sarri, GC Murphy, ME Dieckmann, A Bret, K Quinn, I Kourakis, M Borghesi, LOC Drury and A Ynnerman 2011 New J. Phys. 13073023

[47] T Amano and M Hoshino 2007 Astrophys. J. 661190 